\title{
Application of Matrix Methods On W-Curves
}

\author{
Emre Öztürr*
}

(Communicated by Levent Kula)

\begin{abstract}
In the present study, we find the parametric equations of non-null W-curves through the semi skewsymmetric matrix in three dimensional Lorentz-Minkowski space. Our technic provides more simple but efficient method for find the parametric equations of these curves in comparison to previous studies in mentioned space. Finally, we give some pictures of $\mathrm{W}$-curves in polynomial form.
\end{abstract}

Keywords: W-curve, semi skew-symmetric matrix, Minkowski space.

AMS Subject Classification (2020): Primary: 14H45; Secondary: 40C05; 15A24

\section{Introduction and Preliminaries}

The curve $\gamma$ is called a $W$-curve or curve with constant curvature if it has constant Frenet curvatures. According to relativity theory, the free particles move on these curves in space. One can consider these curves as an integral curve of the helicoidal vector field in Lie algebra or as a solution of the system of linear homogeneous ordinary differential equations of first order with constant (Frenet curvatures) coefficients. These curves widely investigated in Euclidean space ([1], [2]) and in Lorentz-Minkowski space ([5], [6]). The generic parametric equations of unit speed $W$-curves in $\mathbb{R}^{2 n+1}$ Euclidean space and $\mathbb{R}_{1}^{2 n+1}$ Lorentz-Minkowski space are given by

$$
\alpha(s)=a s \overrightarrow{e_{0}}+\sum_{i=1}^{n} r_{i}\left(\cos \left(\mu_{i} s\right) \overrightarrow{e_{2 i-1}}+\sin \left(\mu_{i} s\right) \overrightarrow{e_{2 i}}\right)
$$

and

$$
\begin{aligned}
\gamma(s)= & \left(a_{1} \sinh \lambda_{1} s+b_{1} \cosh \lambda_{1} s, a_{1} \cosh \lambda_{1} s+b_{1} \sinh \lambda_{1} s\right. \\
& a_{2} \sin \lambda_{2} s+b_{2} \cos \lambda_{2} s, a_{2} \cos \lambda_{2} s-b_{2} \sin \lambda_{2} s, \ldots, \\
& \left.a_{n} \sin \lambda_{n} s+b_{n} \cos \lambda_{n} s, a_{n} \cos \lambda_{n} s-b_{n} \sin \lambda_{n} s, c s\right)
\end{aligned}
$$

where $\left\{\overrightarrow{e_{0}}, \overrightarrow{e_{1}}, \ldots, \overrightarrow{e_{2 n}}\right\}$ is orthonormal base of $\mathbb{R}^{2 n+1}$ such that

$$
a^{2}+\sum_{i=1}^{n}\left(r_{i} \mu_{i}\right)^{2}=1
$$

and

$$
-a_{1}^{2} \lambda_{1}^{2}+b_{1}^{2} \lambda_{1}^{2}+c^{2}+\sum_{i=2}^{n}\left(a_{i}^{2}+b_{i}^{2}\right) \lambda_{i}^{2}=\mp 1
$$

respectively. If $a=0$ then $\alpha$ lies on hypersphere, otherwise $\alpha$ fully lies in $\mathbb{R}^{2 n+1}$. If $c=0, \gamma$ lies on $\mathbb{S}_{1}^{2 n}$ pseudosphere or on $\mathbb{H}_{1}^{2 n}$ pseudohyperbolic space otherwise $\gamma$ fully lies in $\mathbb{R}_{1}^{2 n+1}$ ([5], Theorem 3.4). In ([1], Proof of Theorem 1, pp. 460-463), $W$-curves are characterized by chord property with the following condition,

$$
\langle\gamma(t)-\gamma(s), T(t)-T(s)\rangle=0
$$


where $T=\gamma^{\prime}(s)$ is unit tangent vector field of the curve. Öztürk and Yaylı ([5], Definition 3.1) called these curves as $C$-curve and investigated the relations between $W$-curves and this curve in Lorentz-Minkowski space. In [2], these curves characterized by algebraic methods with respect to study of [1]. Walrave [6] classified all of $W$-curves by using some analysis technics in three dimensional Minkowski space. Besides in [5], curves which are satisfy the condition (C) are investigated and given some characterizations about the Frenet vectors of the curve.

The metric tensor (inner product) of Lorentz-Minkowski space is given by

$$
\langle u, v\rangle=-u_{1} v_{1}+\sum_{i=2}^{n} u_{i} v_{i}
$$

where $u=\left(u_{1}, u_{2}, \ldots, u_{n}\right)$ and $v=\left(v_{1}, v_{2}, \ldots, v_{n}\right)$. In Lorentz-Minkowski space, vectors have different causal characters such as if $\langle v, v\rangle>0$ or $v=0,\langle v, v\rangle<0$ and $\langle v, v\rangle=0(v \neq 0)$ then $v$ is called by spacelike, timelike and lightlike (or null) vector respectively. Norm of any vector is given by $\|v\|=\sqrt{|\langle v, v\rangle|}$ [4]. For any regular curve $\gamma: I \rightarrow \mathbb{R}_{1}^{n}$ if $\left\langle\gamma^{\prime}, \gamma^{\prime}\right\rangle>0,\left\langle\gamma^{\prime}, \gamma^{\prime}\right\rangle\left\langle 0\right.$ or $\left\langle\gamma^{\prime}, \gamma^{\prime}\right\rangle=0$ then the curve is called by spacelike, timelike or lightlike (null) curve respectively [3]. If $\left\langle\gamma^{\prime}(s), \gamma^{\prime}(s)\right\rangle=\mp 1$ for all $s \in I$ then $\gamma$ is called by unit speed curve. Frenet-Serret formulae for non-null $\gamma$ curve with $\left\langle\gamma^{\prime \prime}, \gamma^{\prime \prime}\right\rangle \neq 0$ in $\mathbb{R}_{1}^{3}$ are given by

$$
\left(\begin{array}{c}
T^{\prime} \\
N^{\prime} \\
B^{\prime}
\end{array}\right)=\left(\begin{array}{ccc}
0 & \kappa \eta & 0 \\
-\kappa \epsilon & 0 & -\tau \epsilon \eta \\
0 & -\tau \eta & 0
\end{array}\right)\left(\begin{array}{c}
T \\
N \\
B
\end{array}\right)
$$

where $\{T, N, B\}$ is the Frenet frame of the curve, that is $N=\frac{T^{\prime}}{\left\|T^{\prime}\right\|}$ and $B=T \times N$ such that $\langle T, T\rangle=\epsilon$, $\langle N, N\rangle=\eta$ and $\langle B, B\rangle=-\epsilon \eta$. In [5] unit tangent vector field of all $W$-curves (C-curves) characterized in $\mathbb{R}_{1}^{n}$ by the following theorem.

Theorem 1.1. [5] Let $\gamma: I \rightarrow \mathbb{R}_{1}^{n}$ be a unit speed non-null $W$-curve. Unit tangent vector field of $\gamma$ is given by

$$
T(s)=A \gamma(s)+b
$$

where $A$ is $n \times n$ constant semi skew-symmetric matrix, and $b \in \mathbb{R}_{1}^{n}$ is constant column vector.

In this study, we consider the $W$-curves as an integral curve of its unit tangent vector field via Theorem 1.1 and we calculate the parametric equations of these curves by solve some differential equations which are correspond to special cases of the generic semi skew-symmetric matrix in $\mathbb{R}_{1}^{3}$.

\section{Matrix Methods and Elementary Approaches}

In [6], Walrave used MacLaurin expansions and analytic approach for find the $W$-curves in 3 -dimensional Minkowski space. Instead of using approach of Walrave, we prefer the use algebraic method. Here, our technic is more elementary and quite remarkable because of using only one matrix for finding all $W$-curves in $\mathbb{R}_{1}^{3}$. Through the Theorem 1.1 we make some manipulations on the elements of generic semi skew-symmetric matrix. First of all we give the generic form of the semi skew-symmetric matrix in $\mathbb{R}_{1}^{3}$ by

$$
A=\left(\begin{array}{ccc}
0 & \lambda_{1} & \lambda_{2} \\
\lambda_{1} & 0 & -\lambda_{3} \\
\lambda_{2} & \lambda_{3} & 0
\end{array}\right)
$$

where $1 \leq i \leq 3, \lambda_{i} \in \mathbb{R}$. Here we examine the some special cases of (2.1) through the elements of $A$. Let we consider only one $\lambda_{i}$ is zero in the following three case.

Case 1. Let $\lambda_{1}=0$ and $\lambda_{2}, \lambda_{3}$ are non-zero arbitrary constants in (2.1). In this case, semi skew-symmetric matrix and Darboux rotation vector is given by

$$
A_{1}=\left(\begin{array}{ccc}
0 & 0 & \lambda_{2} \\
0 & 0 & -\lambda_{3} \\
\lambda_{2} & \lambda_{3} & 0
\end{array}\right)
$$


and $\vec{w}=\left(\lambda_{3},-\lambda_{2}, 0\right)$ respectively. Through the Theorem 1.1 we consider the $\gamma(t)=\left(\gamma_{1}(t), \gamma_{2}(t), \gamma_{3}(t)\right)$ is unit speed curve and translation vector $b=\left(c_{1}, c_{2}, 0\right)$. Therefore we write

$$
\left(\begin{array}{c}
\gamma_{1}^{\prime}(t) \\
\gamma_{2}^{\prime}(t) \\
\gamma_{3}^{\prime}(t) \\
0
\end{array}\right)=\left(\begin{array}{cccc}
0 & 0 & \lambda_{2} & c_{1} \\
0 & 0 & -\lambda_{3} & c_{2} \\
\lambda_{2} & \lambda_{3} & 0 & 0 \\
0 & 0 & 0 & 0
\end{array}\right)\left(\begin{array}{c}
\gamma_{1}(t) \\
\gamma_{2}(t) \\
\gamma_{3}(t) \\
1
\end{array}\right)
$$

Thus solution of (2.2) is given by

$$
\begin{aligned}
& \gamma_{1}(t)=\frac{\lambda_{2} c_{3}}{\sqrt{\lambda_{2}^{2}-\lambda_{3}^{2}}} e^{\sqrt{\lambda_{2}^{2}-\lambda_{3}^{2}} t}-\frac{\lambda_{2} c_{4}}{\sqrt{\lambda_{2}^{2}-\lambda_{3}^{2}}} e^{-\sqrt{\lambda_{2}^{2}-\lambda_{3}^{2}} t}+\left(c_{1}-\frac{\lambda_{2}^{2} c_{1}+\lambda_{2} \lambda_{3} c_{2}}{\lambda_{2}^{2}-\lambda_{3}^{2}}\right) t+c_{5} \\
& \gamma_{2}(t)=-\frac{\lambda_{3} c_{3}}{\sqrt{\lambda_{2}^{2}-\lambda_{3}^{2}}} e^{\sqrt{\lambda_{2}^{2}-\lambda_{3}^{2}} t}+\frac{\lambda_{3} c_{4}}{\sqrt{\lambda_{2}^{2}-\lambda_{3}^{2}}} e^{-\sqrt{\lambda_{2}^{2}-\lambda_{3}^{2}} t}+\left(c_{2}+\frac{\lambda_{2} \lambda_{3} c_{1}+\lambda_{3}^{2} c_{2}}{\lambda_{2}^{2}-\lambda_{3}^{2}}\right) t+c_{6} \\
& \gamma_{3}(t)=c_{3} e^{\sqrt{\lambda_{2}^{2}-\lambda_{3}^{2}} t}+c_{4} e^{-\sqrt{\lambda_{2}^{2}-\lambda_{3}^{2}} t}-\frac{\lambda_{2} c_{1}+\lambda_{3} c_{2}}{\lambda_{2}^{2}-\lambda_{3}^{2}}
\end{aligned}
$$

where $1 \leq i \leq 6, c_{i} \in \mathbb{R}$ and $\lambda_{2}^{2}-\lambda_{3}^{2}>0$.

Case 2. Let $\lambda_{2}=0$ and $\lambda_{1}, \lambda_{3}$ are non-zero arbitrary constants in (2.1). In this case, semi skew-symmetric matrix and Darboux rotation vector is given by

$$
A_{2}=\left(\begin{array}{ccc}
0 & \lambda_{1} & 0 \\
\lambda_{1} & 0 & -\lambda_{3} \\
0 & \lambda_{3} & 0
\end{array}\right)
$$

and $\vec{w}=\left(\lambda_{3}, 0, \lambda_{1}\right)$ respectively. Hence we have

$$
\left(\begin{array}{c}
\gamma_{1}^{\prime}(t) \\
\gamma_{2}^{\prime}(t) \\
\gamma_{3}^{\prime}(t) \\
0
\end{array}\right)=\left(\begin{array}{cccc}
0 & \lambda_{1} & 0 & c_{1} \\
\lambda_{1} & 0 & -\lambda_{3} & 0 \\
0 & \lambda_{3} & 0 & c_{2} \\
0 & 0 & 0 & 0
\end{array}\right)\left(\begin{array}{c}
\gamma_{1}(t) \\
\gamma_{2}(t) \\
\gamma_{3}(t) \\
1
\end{array}\right)
$$

Solution of (2.3) is given by

$$
\begin{aligned}
& \gamma_{1}(t)=\frac{\lambda_{1} c_{3}}{\sqrt{\lambda_{1}^{2}-\lambda_{3}^{2}}} e^{\sqrt{\lambda_{2}^{2}-\lambda_{3}^{2}} t}-\frac{\lambda_{1} c_{4}}{\sqrt{\lambda_{1}^{2}-\lambda_{3}^{2}}} e^{-\sqrt{\lambda_{2}^{2}-\lambda_{3}^{2}} t}+\left(c_{1}-\frac{\lambda_{1}^{2} c_{1}-\lambda_{1} \lambda_{3} c_{2}}{\lambda_{1}^{2}-\lambda_{3}^{2}}\right) t+c_{5} \\
& \gamma_{2}(t)=c_{3} e^{\sqrt{\lambda_{1}^{2}-\lambda_{3}^{2}} t}+c_{4} e^{-\sqrt{\lambda_{1}^{2}-\lambda_{3}^{2}} t}-\frac{\lambda_{1} c_{1}-\lambda_{3} c_{2}}{\lambda_{1}^{2}-\lambda_{3}^{2}} \\
& \gamma_{3}(t)=\frac{\lambda_{3} c_{3}}{\sqrt{\lambda_{1}^{2}-\lambda_{3}^{2}}} e^{\sqrt{\lambda_{1}^{2}-\lambda_{3}^{2}} t}-\frac{\lambda_{3} c_{4}}{\sqrt{\lambda_{1}^{2}-\lambda_{3}^{2}}} e^{-\sqrt{\lambda_{1}^{2}-\lambda_{3}^{2}} t}+\left(c_{2}-\frac{\lambda_{1} \lambda_{3} c_{1}-\lambda_{3}^{2} c_{2}}{\lambda_{1}^{2}-\lambda_{3}^{2}}\right) t+c_{6}
\end{aligned}
$$

where $1 \leq i \leq 6, c_{i} \in \mathbb{R}$ and $\lambda_{1}^{2}-\lambda_{3}^{2}>0$.

Case 3. Let $\lambda_{3}=0$ and $\lambda_{1}, \lambda_{2}$ are non-zero arbitrary constants in (2.1). In this case, semi skew-symmetric matrix and Darboux rotation vector is given by

$$
A_{3}=\left(\begin{array}{ccc}
0 & \lambda_{1} & \lambda_{2} \\
\lambda_{1} & 0 & 0 \\
\lambda_{2} & 0 & 0
\end{array}\right)
$$

and $\vec{w}=\left(0,-\lambda_{2}, \lambda_{1}\right)$ respectively. Hence we have

$$
\left(\begin{array}{c}
\gamma_{1}^{\prime}(t) \\
\gamma_{2}^{\prime}(t) \\
\gamma_{3}^{\prime}(t) \\
0
\end{array}\right)=\left(\begin{array}{cccc}
0 & \lambda_{1} & \lambda_{2} & 0 \\
\lambda_{1} & 0 & 0 & c_{1} \\
\lambda_{2} & 0 & 0 & c_{2} \\
0 & 0 & 0 & 0
\end{array}\right)\left(\begin{array}{c}
\gamma_{1}(t) \\
\gamma_{2}(t) \\
\gamma_{3}(t) \\
1
\end{array}\right)
$$


Solution of (2.4) is given by

$$
\begin{aligned}
& \gamma_{1}(t)=c_{3} e^{\sqrt{\lambda_{1}^{2}+\lambda_{2}^{2}} t}+c_{4} e^{-\sqrt{\lambda_{1}^{2}+\lambda_{2}^{2}} t}-\frac{\lambda_{1} c_{1}+\lambda_{2} c_{2}}{\lambda_{1}^{2}+\lambda_{2}^{2}} \\
& \gamma_{2}(t)=\frac{\lambda_{1} c_{3}}{\sqrt{\lambda_{1}^{2}+\lambda_{2}^{2}}} e^{\sqrt{\lambda_{1}^{2}+\lambda_{2}^{2}} t}-\frac{\lambda_{1} c_{4}}{\sqrt{\lambda_{1}^{2}+\lambda_{2}^{2}}} e^{-\sqrt{\lambda_{1}^{2}+\lambda_{2}^{2}} t}+\left(c_{1}-\frac{\lambda_{1}^{2} c_{1}+\lambda_{1} \lambda_{2} c_{2}}{\lambda_{1}^{2}+\lambda_{2}^{2}}\right) t+c_{5} \\
& \gamma_{3}(t)=\frac{\lambda_{2} c_{3}}{\sqrt{\lambda_{1}^{2}+\lambda_{2}^{2}}} e^{\sqrt{\lambda_{1}^{2}+\lambda_{2}^{2}} t}-\frac{\lambda_{2} c_{4}}{\sqrt{\lambda_{1}^{2}+\lambda_{2}^{2}}} e^{-\sqrt{\lambda_{1}^{2}+\lambda_{2}^{2}} t}+\left(c_{2}-\frac{\lambda_{1} \lambda_{2} c_{1}+\lambda_{2}^{2} c_{2}}{\lambda_{1}^{2}+\lambda_{2}^{2}}\right) t+c_{6}
\end{aligned}
$$

where $1 \leq i \leq 6, c_{i} \in \mathbb{R}$.

Now we examine the special conditions of above cases.

Case 4. Let $\lambda_{1}=0$ and $\lambda_{2}=\lambda, \lambda_{3}=|\lambda|$ are non-zero arbitrary constants in (2.1). In this case, semi skewsymmetric matrix and Darboux rotation vector is given by

$$
A_{4}=\left(\begin{array}{ccc}
0 & 0 & \lambda \\
0 & 0 & -|\lambda| \\
\lambda & |\lambda| & 0
\end{array}\right)
$$

and $\vec{w}=(|\lambda|,-\lambda, 0)$ respectively. Hence we have

$$
\left(\begin{array}{c}
\gamma_{1}^{\prime}(t) \\
\gamma_{2}^{\prime}(t) \\
\gamma_{3}^{\prime}(t) \\
0
\end{array}\right)=\left(\begin{array}{cccc}
0 & 0 & \lambda & c_{1} \\
0 & 0 & -|\lambda| & c_{2} \\
\lambda & |\lambda| & 0 & 0 \\
0 & 0 & 0 & 0
\end{array}\right)\left(\begin{array}{c}
\gamma_{1}(t) \\
\gamma_{2}(t) \\
\gamma_{3}(t) \\
1
\end{array}\right)
$$

Solution of (2.5) is given by

$$
\begin{aligned}
& \gamma_{1}(t)=\frac{\lambda^{2}}{6}\left(c_{1}+\operatorname{sgn}(\lambda) c_{2}\right) t^{3}+\left(\lambda c_{3}+c_{1}\right) t+c_{4} \\
& \gamma_{2}(t)=-\frac{\lambda^{2}}{6}\left(\operatorname{sgn}(\lambda) c_{1}+c_{2}\right) t^{3}+\left(c_{2}-|\lambda| c_{3}\right) t+c_{5} \\
& \gamma_{3}(t)=\frac{1}{2}\left(\lambda c_{1}+|\lambda| c_{2}\right) t^{2}+c_{3}
\end{aligned}
$$

where $c_{1} \in \mathbb{R}^{>0}, 2 \leq i \leq 5, c_{i} \in \mathbb{R}$ and

$$
\operatorname{sgn}(\lambda)=\left\{\begin{array}{cc}
1 & , \quad \lambda>0 \\
-1, & \lambda<0
\end{array}\right.
$$

Case 5. By similar procedures above we take $\lambda_{2}=0$ and $\lambda_{1}=\lambda, \lambda_{3}=|\lambda|$ and we get

$$
\begin{aligned}
& \gamma_{1}(t)=\frac{1}{6} \lambda\left(\lambda c_{1}-|\lambda| c_{2}\right) t^{3}+\left(\lambda c_{3}+c_{1}\right) t+c_{4} \\
& \gamma_{2}(t)=\frac{1}{2}\left(\lambda c_{1}-|\lambda| c_{2}\right) t^{2}+c_{3} \\
& \gamma_{3}(t)=\frac{1}{6}|\lambda|\left(\lambda c_{1}-|\lambda| c_{2}\right) t^{3}+\left(|\lambda| c_{3}+c_{2}\right) t+c_{5}
\end{aligned}
$$

where $c_{1} \in \mathbb{R}^{>0}$ and $2 \leq i \leq 5, c_{i} \in \mathbb{R}$.

Case 6. If we take $\lambda_{3}=0$ and $\lambda_{1}=\lambda, \lambda_{2}=|\lambda|$ we get

$$
\begin{aligned}
& \gamma_{1}(t)=c_{3} e^{\sqrt{2}|\lambda| t}+c_{4} e^{-\sqrt{2}|\lambda| t}-\frac{c_{1}+\operatorname{sgn}(\lambda) c_{2}}{2 \lambda} \\
& \gamma_{2}(t)=\frac{\operatorname{sgn}(\lambda)}{\sqrt{2}}\left(c_{3} e^{\sqrt{2}|\lambda| t}-c_{4} e^{-\sqrt{2}|\lambda| t}\right)+\frac{c_{1}-\operatorname{sgn}(\lambda) c_{2}}{2} t+c_{5} \\
& \gamma_{3}(t)=\frac{\operatorname{sgn}(\lambda)}{\sqrt{2}}\left(c_{3} e^{\sqrt{2}|\lambda| t}-c_{4} e^{-\sqrt{2}|\lambda| t}\right)+\frac{c_{2}-\operatorname{sgn}(\lambda) c_{1}}{2} t+c_{6}
\end{aligned}
$$

where $c_{1} \in \mathbb{R}^{>0}$ and $2 \leq i \leq 6, c_{i} \in \mathbb{R}$. 
So far we considered only one $\lambda_{i}$ is zero at previous cases. Now we take two of $\lambda_{i}$ is zero in the following cases.

Case 7. Let $\lambda_{2}=\lambda_{3}=0$ and $\lambda_{1}=\lambda \neq 0$ in (2.1). In this case, semi skew-symmetric matrix and Darboux rotation vector is given by

$$
A_{5}=\left(\begin{array}{ccc}
0 & \lambda & 0 \\
\lambda & 0 & 0 \\
0 & 0 & 0
\end{array}\right)
$$

and $\vec{w}=(0,0, \lambda)$ respectively. Obviously we have

$$
\left(\begin{array}{c}
\gamma_{1}^{\prime}(t) \\
\gamma_{2}^{\prime}(t) \\
\gamma_{3}^{\prime}(t) \\
0
\end{array}\right)=\left(\begin{array}{cccc}
0 & \lambda & 0 & 0 \\
\lambda & 0 & 0 & 0 \\
0 & 0 & 0 & c \\
0 & 0 & 0 & 0
\end{array}\right)\left(\begin{array}{c}
\gamma_{1}(t) \\
\gamma_{2}(t) \\
\gamma_{3}(t) \\
1
\end{array}\right)
$$

The generic solution of above differential equation is given by

$$
\begin{aligned}
& \gamma_{1}(t)=a_{1} \cosh \lambda t+a_{2} \sinh \lambda t \\
& \gamma_{2}(t)=a_{1} \sinh \lambda t+a_{2} \cosh \lambda t \\
& \gamma_{3}(t)=c t
\end{aligned}
$$

where $a_{1}, a_{2} \in \mathbb{R}$. Let we give the type of the curves which are correspond to special case of (2.6).

Theorem 2.1. Unit speed curve $\gamma(s)=\left(\gamma_{1}(s), \gamma_{2}(s), \gamma_{3}(s)\right)$ in the form of (2.6) is given by one of the following cases.

1) If $a_{2}=0$ and $a_{1}=a \neq 0$ then

$$
\gamma(s)=\left(a \cosh \frac{\lambda}{u} s, a \sinh \frac{\lambda}{u} s, \frac{c}{u} s\right)
$$

is a spacelike hyperbolic helix with timelike normal where $u=\sqrt{c^{2}+a^{2} \lambda^{2}}$.

2) If $a_{1}=0$ and $a_{2}=a \neq 0$ then one of the following cases occur.

i) If $c^{2}-a^{2} \lambda^{2}>0$ then

$$
\gamma(s)=\left(a \sinh \frac{\lambda}{u} s, a \cosh \frac{\lambda}{u} s, \frac{c}{u} s\right)
$$

is a spacelike hyperbolix helix with spacelike normal where $u=\sqrt{c^{2}-a^{2} \lambda^{2}}$.

ii) If $c^{2}-a^{2} \lambda^{2}<0$ then

$$
\gamma(s)=\left(a \sinh \frac{\lambda}{u} s, a \cosh \frac{\lambda}{u} s, \frac{c}{u} s\right)
$$

is a timelike hyperbolic helix where $u=\sqrt{a^{2} \lambda^{2}-c^{2}}$.

Proof. 1) Suppose that $a_{2}=0$ and $a_{1}=a \neq 0$. Due to (2.6) we write $\gamma(t)=(a \cosh \lambda t, a \sinh \lambda t, c t)$. Thus

$$
\left\langle\gamma^{\prime}(t), \gamma^{\prime}(t)\right\rangle=c^{2}+a^{2} \lambda^{2}>0
$$

and

$$
\left\langle\gamma^{\prime \prime}(t), \gamma^{\prime \prime}(t)\right\rangle=-a^{2} \lambda^{4}<0
$$

So $\gamma$ is spacelike curve with timelike normal. If we consider $\left\|\gamma^{\prime}(t)\right\|=\sqrt{c^{2}+a^{2} \lambda^{2}}=u$ then we get unit speed curve

$$
\gamma(s)=\left(a \cosh \frac{\lambda}{u} s, a \sinh \frac{\lambda}{u} s, \frac{c}{u} s\right)
$$

Besides the curvature and torsion of this curve $\kappa=\frac{a \lambda^{2}}{u^{2}}$ and $\tau=\frac{c \lambda}{u^{2}}$ are constant respectively. Inner product of unit tangent vector field $T$ of $\gamma$ and $\vec{v}=(0,0,1)$

$$
\langle T, \vec{v}\rangle=\frac{c}{u}
$$


is constant so $\vec{v}=(0,0,1)$ is the directrix of the rotation axis of this curve.

2) Suppose that $a_{1}=0$ and $a_{2}=a \neq 0$. According to (2.6) we write $\gamma(t)=(a \sinh \lambda t, a \cosh \lambda t, c t)$ and $\left\langle\gamma^{\prime}(t), \gamma^{\prime}(t)\right\rangle=c^{2}-a^{2} \lambda^{2}$.

i) Let $\left\langle\gamma^{\prime}(t), \gamma^{\prime}(t)\right\rangle=c^{2}-a^{2} \lambda^{2}>0$. Therefore $\left\langle\gamma^{\prime \prime}(t), \gamma^{\prime \prime}(t)\right\rangle=a^{2} \lambda^{4}>0$ and $\gamma$ is spacelike curve with spacelike normal. If we consider $\left\|\gamma^{\prime}(t)\right\|=\sqrt{c^{2}-a^{2} \lambda^{2}}=u$ then we get unit speed curve

$$
\gamma(s)=\left(a \sinh \frac{\lambda}{u} s, a \cosh \frac{\lambda}{u} s, \frac{c}{u} s\right)
$$

By straightforward calculations we get $\kappa=\frac{a \lambda^{2}}{u^{2}}$ and $\tau=\frac{c \lambda}{u^{2}}$ are constant and $|\tau|>\kappa$. Similar to above we find the directrix of the rotation axis of the curve as $\vec{v}=(0,0,1)$.

ii) Let $\left\langle\gamma^{\prime}(t), \gamma^{\prime}(t)\right\rangle=c^{2}-a^{2} \lambda^{2}<0$. If we consider $\left\|\gamma^{\prime}(t)\right\|=\sqrt{a^{2} \lambda^{2}-c^{2}}=u$ then we get the equation of the unit speed timelike curve as

$$
\gamma(s)=\left(a \sinh \frac{\lambda}{u} s, a \cosh \frac{\lambda}{u} s, \frac{c}{u} s\right)
$$

and $\kappa=\frac{a \lambda^{2}}{u^{2}}$ and $\tau=-\frac{c \lambda}{u^{2}}$ are constant such that $|\tau|<\kappa$. Obviously $\vec{v}=(0,0,1)$ is the directrix of the rotation axis of the curve.

Case 8. Let $\lambda_{1}=\lambda_{3}=0$ and $\lambda_{2}=\lambda \neq 0$ in (2.1). In this case, semi skew-symmetric matrix and Darboux rotation vector is given by

$$
A_{6}=\left(\begin{array}{lll}
0 & 0 & \lambda \\
0 & 0 & 0 \\
\lambda & 0 & 0
\end{array}\right)
$$

and $\vec{w}=(0,-\lambda, 0)$ respectively. Hence we have

$$
\left(\begin{array}{c}
\gamma_{1}^{\prime}(t) \\
\gamma_{2}^{\prime}(t) \\
\gamma_{3}^{\prime}(t) \\
0
\end{array}\right)=\left(\begin{array}{cccc}
0 & 0 & \lambda & 0 \\
0 & 0 & 0 & c \\
\lambda & 0 & 0 & 0 \\
0 & 0 & 0 & 0
\end{array}\right)\left(\begin{array}{c}
\gamma_{1}(t) \\
\gamma_{2}(t) \\
\gamma_{3}(t) \\
1
\end{array}\right)
$$

Thus solution of the above differential equation is given by

$$
\begin{aligned}
& \gamma_{1}(t)=\operatorname{sgn}(\lambda) c_{1} e^{|\lambda| t}-\operatorname{sgn}(\lambda) c_{2} e^{-|\lambda| t}+c_{3} \\
& \gamma_{2}(t)=c s+d \\
& \gamma_{3}(t)=c_{1} e^{|\lambda| t}+c_{2} e^{-|\lambda| t}
\end{aligned}
$$

where $1 \leq i \leq 3, c_{i}, d \in \mathbb{R}$.

Case 9. Let $\lambda_{1}=\lambda_{2}=0$ and $\lambda_{3}=\lambda \neq 0$ in (2.1). In this case, semi skew-symmetric matrix and Darboux rotation vector is given by

$$
A_{7}=\left(\begin{array}{ccc}
0 & 0 & 0 \\
0 & 0 & -\lambda \\
0 & \lambda & 0
\end{array}\right)
$$

and $\vec{w}=(\lambda, 0,0)$ respectively. By similar previous calculations we get the generic solution of $\gamma(t)=$ $\left(\gamma_{1}(t), \gamma_{2}(t), \gamma_{3}(t)\right)$ as

$$
\begin{aligned}
& \gamma_{1}(t)=c t \\
& \gamma_{2}(t)=a \cos \lambda t \\
& \gamma_{3}(t)=a \sin \lambda t
\end{aligned}
$$

where $a \in \mathbb{R}$. Thus $\left\langle\gamma^{\prime}(t), \gamma^{\prime}(t)\right\rangle=a^{2} \lambda^{2}-c^{2}$. Depends on to sign we have the following theorem.

Theorem 2.2. Unit speed curve $\gamma(s)=\left(\gamma_{1}(s), \gamma_{2}(s), \gamma_{3}(s)\right)$ in the form of (2.7) is given by one of the following cases. 
1) If $a^{2} \lambda^{2}-c^{2}>0$ then

$$
\gamma(s)=\left(\frac{c}{u} s, a \cos \frac{\lambda}{u} s, a \sin \frac{\lambda}{u} s\right)
$$

is a spacelike circular helix where $u=\sqrt{a^{2} \lambda^{2}-c^{2}}$.

2) If $a^{2} \lambda^{2}-c^{2}<0$ then

$$
\gamma(s)=\left(\frac{c}{u} s, a \cos \frac{\lambda}{u} s, a \sin \frac{\lambda}{u} s\right)
$$

is timelike circular helix where $u=\sqrt{c^{2}-a^{2} \lambda^{2}}$.

Proof. 1) By straightforward calculations, the parametric equation, curvature and the torsion of this curve is given by $\gamma(s)=\left(\frac{c}{u} s, a \cos \frac{\lambda}{u} s, a \sin \frac{\lambda}{u} s\right), \kappa=a\left(\frac{\lambda}{u}\right)^{2}$ and $\tau=c \frac{\lambda}{u^{2}}$ respectively. Besides $|\tau|<\kappa$ and $\vec{v}=(1,0,0)$ is the directrix of the rotation axis of the curve.

2) Similary the parametric equation, curvature and the torsion of this curve is given by $\gamma(s)=$ $\left(\frac{c}{u} s, a \cos \frac{\lambda}{u} s, a \sin \frac{\lambda}{u} s,\right), \kappa=a\left(\frac{\lambda}{u}\right)^{2}$ and $\tau=c \frac{\lambda}{u^{2}}$ respectively where $a>0$. Besides $\kappa<|\tau|$ and $\vec{v}=(1,0,0)$ is the directrix of the rotation axis of the curve.

Example 2.1. Let we give the curve $\gamma: I \rightarrow \mathbb{R}_{1}^{3}$ by

$$
\gamma(s)=\left(\frac{1}{6} \lambda|\lambda| s^{3},-\frac{1}{6} \lambda^{2} s^{3}+s, \frac{1}{2}|\lambda| s^{2}\right)
$$

Obviously this curve is a unit speed spacelike $W$-curve. It can be easily seen that

$$
\langle\gamma(t)-\gamma(s), T(t)-T(s)\rangle=0
$$

where $T$ is the unit tangent vector field of the curve. For $\lambda=1$, this curve is illustared by Figure 1 (a).

Example 2.2. Let we give the curve $\gamma: I \rightarrow \mathbb{R}_{1}^{3}$ by

$$
\gamma(s)=\left(\frac{1}{6} \lambda^{2} s^{3}+s, \frac{1}{2} \lambda s^{2}, \frac{1}{6} \lambda|\lambda| s^{3}\right)
$$

This curve is a unit speed timelike $W$-curve. It can be easily seen that

$$
\langle\gamma(t)-\gamma(s), T(t)-T(s)\rangle=0
$$

where $T$ is the unit tangent vector field of the curve. For $\lambda=1$, this curve is illustared by Figure $1(b)$.

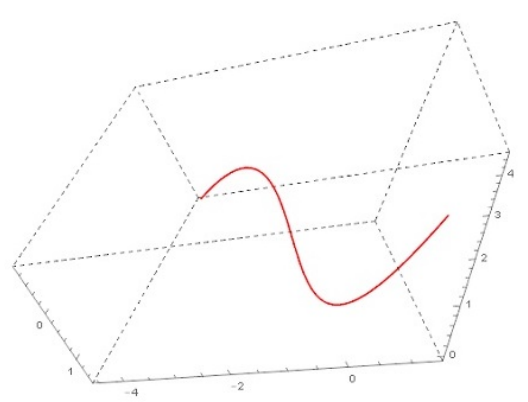

(a) Spacelike W-Curve

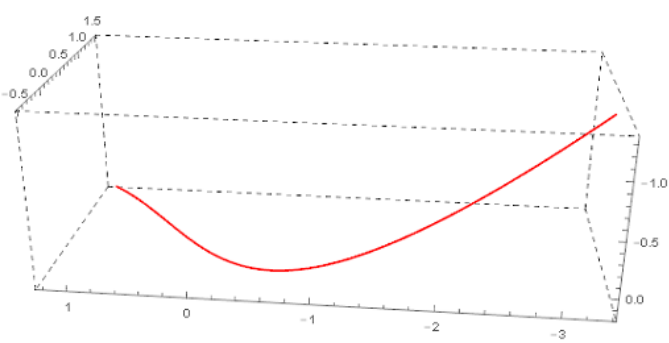

(b) Timelike W-Curve

Figure 1. Semicubic W-Curves 


\section{Conclusions}

This study provides algebraic method for classify the $W$-curves by using only one semi skew-symmetric matrix in three dimensional Lorentz-Minkowski space. Obviously this approach more elementary and more useful in comparison to previous studies. One can obtain these curves in high dimensions or in different spaces (whose have different metric tensor) by using the same approach.

\section{References}

[1] Chen, B. Y., Kim, D. S., Kim, Y. H.: New Characterizations of W-Curves. Publ. Math. Debrecen. 69 (4), $457-472$ (2006).

[2] Kim, D. S., Kim, Y. H.: New Characterizations of Spheres, Cylinders and W-Curves. Linear Algebra and Its Applications. 432 (11), $3002-3006$ (2010).

[3] Kühnel, W.: Differential Geometry Curves-Surfaces-Manifolds. American Mathematical Society. USA (2006).

[4] O'Neill, B.: Semi-Riemann Geometry with Applications to Relativity. Academic Press. Inc. London (1983).

[5] Öztürk, E., Yaylı, Y.: W-Curves In Lorentz-Minkowski Space. Mathematical Sciences and Applications E-Notes. 5 (2), 76-88 (2017).

[6] Walrave, J.: Curves and Surfaces in Minkowski Space. Ph.D. thesis. K. U. Leuven Fac. of Science, Leuven (1995).

\section{Affiliations}

EMRE ÖZTÜRK

AdDress: Turkish Court of Accounts, 06520, Ankara-Turkey.

E-MAIL: emreozturk1471@gmail.com

ORCID ID: 0000-0001-6638-3233 